\title{
Hepatic Steatosis and Fibrosis in Type 2 Diabetes: A Risk- Based Approach to Targeted Screening
}

\author{
Hossein Poustchi, MD, PhD'; Fariba Alaei-Shahmiri, MD, PhD²; Rokhsareh Aghili, MD, PhD²; Sohrab Nobarani, MD²; Mojtaba Malek, MD³; \\ Mohammad E. Khamseh, MD² \\ ${ }^{1}$ Liver and Pancreatobiliary Diseases Research Center, Digestive Disease Research Institute, Shariati Hospital, Tehran University of Medical \\ Sciences, Tehran, Iran \\ ${ }^{2}$ Endocrine Research Center, Institute of Endocrinology and Metabolism, Iran University of Medical Sciences, Tehran, Iran \\ ${ }^{3}$ Research Center for Prevention of Cardiovascular Disease, Institute of Endocrinology and Metabolism, Iran University of Medical Sciences, \\ Tehran, Iran
}

\begin{abstract}
Background: Non-alcoholic fatty liver disease (NAFLD) is common in people with type 2 diabetes mellitus (T2DM). We aimed to explore predictive factors of NAFLD in T2DM and identify high risk subgroups.

Methods: This was a cross-sectional study including 100 individuals with T2DM and 100 without diabetes matched for age, sex, and body mass index (BMI). Hepatic steatosis grades (calculated by controlled attenuation parameters-CAP score-3), and liver fibrosis stages (F0-F4) were determined using transient elastography.

Results: The frequency of NAFLD was comparable between the two study groups. However, CAP scores were significantly higher in individuals with diabetes $(294.90 \pm 53.12$ vs. $269.78 \pm 45.05 \mathrm{~dB} / \mathrm{m} ; P<0.001)$. Fifty percent of individuals with diabetes had severe steatosis (S3), while this figure was $31.6 \%$ in those without diabetes $(P<0.05)$. Significant fibrosis (F2-F4) was more frequent in individuals with T2DM (13\% vs. $4.1 \%, P=0.02)$. Individuals with T2DM and advanced fibrosis had significantly higher $\mathrm{BMI}$, waist circumference (WC), waist-hip ratio (WHR), alanine aminotransferase (ALT), aspartate aminotransferase (AST), and CAP score compared to those without fibrosis $(P<0.05)$. In the regression analysis, a model including BMI, WHR, AST and female gender explained $50 \%$ of the variation in CAP score in patients with diabetes (all $P<0.05$, adjusted $\mathrm{R}^{2}: 0.508$ ). CAP scores were also the major determinant of liver fibrosis in this group (OR: 1.04; $\mathrm{Cl}: 1.017-1.063 ; P=0.001)$.

Conclusion: Individuals with diabetes are more likely to have severe fibrosis. Obesity (especially central obesity), the female gender, elevated liver enzymes, and higher degree of insulin resistance are associated with more advanced liver disease in individuals with T2DM.

Keywords: Diabetes mellitus, Hepatic steatosis, Liver fibrosis, NAFLD, Non-alcoholic fatty liver disease, Type 2

Cite this article as: Poustchi H, Alaei-Shahmiri F, Aghili R, Nobarani S, Malek M, Khamseh ME. Hepatic steatosis and fibrosis in type 2 diabetes: a risk-based approach to targeted screening. Arch Iran Med. 2021;24(3):177-186. doi: 10.34172/aim.2021.28.

Received: April 20, 2020, Accepted: October 19, 2020, ePublished: March 1, 2021
\end{abstract}

\section{Introduction}

Non-alcoholic fatty liver disease (NAFLD) is a common chronic liver disease, encompassing a range of histopathologic abnormalities from simple steatosis to advanced fibrosis and cirrhosis. ${ }^{1}$ The reported prevalence of NAFLD in the general population varies from $19 \%$ to $46 \%$ based on the study population, age, and the tools used for diagnosis. ${ }^{2}$ Based on a meta-analysis published in 2016, NAFLD is estimated to affect approximately one third of the Iranian population (33.9\%, 95\% CI: $26.4 \%-$ $41.5 \%){ }^{2}$

It is well known that NAFLD is associated with obesity, type 2 diabetes (T2DM), and insulin resistance. ${ }^{3}$ On the other hand, diabetes is an independent risk factor of advanced fibrosis in patients with NAFLD. ${ }^{4}$ In addition, the incidence of cirrhosis is higher in patients with diabetes. ${ }^{5}$ With the steady increase in the prevalence of T2DM, the burden of NAFLD is accordingly expected to increase. Therefore, early diagnosis and management are required to prevent progressive liver disease.

Routine screening of steatosis and liver fibrosis in patients with diabetes is not generally recommended due to the lack of precise and non-invasive methods for diagnosis of liver fibrosis, as well as limited therapies available. ${ }^{6}$ While liver biopsy is considered to be the gold standard method of liver disease diagnosis, it is not routinely performed for NAFLD detection due to its invasive nature. Currently, ultrasound is the most commonly used tool for detecting fatty liver. However, abdominal ultrasound is not susceptible to mild steatosis. ${ }^{7}$ Computed tomography (CT) scan also has a low sensitivity in detecting earlier stages of fatty liver, while at the same time exposing patients to radiation. Although magnetic resonance imaging (MRI) is considered to be the most accurate quantitative method to measure liver fat, its high cost is an important barrier for routine use in clinical practice.

Fibroscan is a non-invasive tool that measures hepatic elasticity using ultrasound transient elastography (TE). ${ }^{8-10}$ 
With a sensitivity of $94.80 \%$ and specificity of $77.08 \%$, $\mathrm{TE}$ can be used as an acceptable noninvasive screening tool in large populations. ${ }^{11}$ Given that liver stiffness is correlated to liver fibrosis, TE can be used to detect liver cirrhosis with high accuracy. ${ }^{12}$ The fibroscan device has different sized probes for various body sizes. To overcome body weight as a potential barrier in obese individuals, an $\mathrm{XL}$ probe as well as a controlled attenuation parameter (CAP) can be implemented on the device to measure the level of steatosis. ${ }^{13}$

Existing evidence indicates that the prevalence, severity and outcomes of NAFLD could be influenced by some underlying factors including ethnicity. ${ }^{14}$ To the best of our knowledge, there is currently no study on liver steatosis and fibrosis using fibroscan in individuals with T2DM compared to those without diabetes matched for age, sex, and body mass index (BMI) in the Iranian population. Considering NAFLD as a multisystem disease affecting extra-hepatic organs and its association with hard endpoints such as cardiovascular disease, we aimed to explore the frequency and predictors of NAFLD in individuals with T2DM and to identify high risk subgroups.

\section{Materials and Methods}

This was a cross-sectional study including 100 individuals with T2DM and 100 without diabetes matched for age, sex, and BMI by category/mean matching method.

The study included patients 30 to 65 years of age who had diabetes for at least 3 years. The following exclusion criteria were applied: history of liver disease or symptoms of active liver disease, evidence of other chronic liver diseases such as $\mathrm{HBS} \mathrm{Ag}^{+}$, Anti-HCV $\mathrm{Ab}^{+}$, history of alpha-1 anti-trypsin deficiency, autoimmune hepatitis, liver damage caused by drugs, primary biliary cirrhosis, primary sclerosing cholangitis, biliary obstruction in previous radiological studies, advanced complications of diabetes (CKD $\geq$ Stage III, CHF, Advanced CVD), excessive alcohol consumption ( $>20 \mathrm{~g} / \mathrm{d})$, pregnancy, and intake of medications that can lead to NAFLD, namely, corticosteroids, tamoxifen, amiodarone and methotrexate.

\section{Sample Size Calculation}

Sample size was determined using the $\mathrm{G}^{*}$ Power software. Considering the prevalence of liver fibrosis in individuals with diabetes (40.3\%) and non-diabetic people (17\%) reported in previous studies, ${ }^{15}$ a sample of 160 subjects (80 in each groups) could provide sufficient power (80\%) at the $5 \%$ significance level. However, a total of 200 subjects were recruited to increase the study power. Furthermore, given previous studies suggesting 10 subjects per variable as an appropriate sample size for linear and logistic regression models, ${ }^{16,17}$ the determined sample size would be enough to ensure the evaluation of main predictors of liver steatosis and fibrosis in the study population (19 candidate variables in overall participants and 9 candidate variables in each study group, as described in the Statistical Analysis section).

\section{Clinical Assessment}

Demographic and anthropometric measurements were obtained by a trained investigator. BMI was calculated by dividing body weight (in kilograms) by the square of the height (in meters). A fasting blood sample was taken for the measurement of metabolic variables including fasting blood sugar (FBS), glycosylated hemoglobin (HbA1c), triglyceride (TG), total cholesterol (Chol), high density lipoprotein (HDL), and low-density lipoprotein (LDL), aspartate aminotransferase (AST), alanine aminotransferase (ALT), gamma-Glutamyl transpeptidase (GGT), alkaline phosphatase (ALP), insulin level, and viral markers (HBS $\mathrm{Ag}$ and Anti- HCV). Insulin resistance was assessed by the homeostatic model assessment - insulin resistance (HOMA-IR) based on the following formula: [fasting glucose $(\mathrm{mg} / \mathrm{dL}) \times$ insulin $(\mu \mathrm{U} / \mathrm{mL})]$ divided by $405 .{ }^{18}$

Blood glucose levels were determined by the enzymatic colorimetric method. Enzymatic assays were also used to determine the levels of serum triglyceride, total cholesterol and HDL-cholesterol. All analyses were performed using Pars Azmun diagnostic kits (Pars Azmun Co., Tehran, Iran) with a between- and within-run coefficient of variation $<6.2 \%$. LDL-cholesterol was calculated using a modified version of the Friedewald equation. ${ }^{19}$ Serum insulin (ECLIA method), ALT (IFCC method), AST (IFCC without pyridoxal phosphate activation), GGT (acc.Ver.1 Standardized against IFCC) and AlP (acc. IFCC Gen2 ALP2L) were determined using Roche Diagnostics kits (Roche Cobas 6000 analyzer) with coefficient of variations $<3.2 \%$.

Dietary intake was assessed using a food frequency questionnaire (FFQ), which was completed for all participants during a face-to-face interview. To assess physical activity, the short form of the international physical activity questionnaire (IPAQ-SF) was used categorizing physical activity levels as low, moderate, and high. ${ }^{20}$

All TE was performed using FibroScan ${ }^{\circledR}$ (Echosens, Paris, France) by a trained specialist physician who was blinded to the subjects. Participants were advised to be fasted for at least 3 hours prior to their TE test. For each participant, at least 10 valid measurements were obtained, with an interquartile range to median ratio $<0.3$. The final score was reported as the median of individual measurements. Based on the measurements, a CAP score (amount of steatosis in decibels per meter $(\mathrm{dB} / \mathrm{m})$ of liver tissue) was designated to each individual, from which NAFLD diagnosis and the grade of steatosis were determined. Steatosis can range from 100 to $400 \mathrm{~dB} / \mathrm{m}$; individuals with a score greater than $238 \mathrm{~dB} / \mathrm{m}$ were diagnosed with NAFLD, with the following CAP score ranges, indicating the grade of steatosis: grade 1 (S1): $238 \mathrm{~dB} / \mathrm{m} \leq \mathrm{a}$ CAP 
score $\leq 259 \mathrm{~dB} / \mathrm{m}$, grade $2(\mathrm{~S} 2): 260 \mathrm{~dB} / \mathrm{m} \leq$ CAP score $\leq$ $291 \mathrm{~dB} / \mathrm{m}$, and grade 3 (S3): $292 \mathrm{~dB} / \mathrm{m} \leq$ CAP score. ${ }^{21,22}$

Hepatic fibrosis and its stages were assessed by the METAVIR scoring system. The five stages of hepatic fibrosis include: F0: no fibrosis: mild fibrosis (F1 $<7.1$ $\mathrm{KPa})$, significant fibrosis $(7.1 \leq \mathrm{F} 2<9.5 \mathrm{kPa})$, severe fibrosis $(9.5 \leq \mathrm{F} 3<12.5 \mathrm{kPa})$, cirrhosis $(\mathrm{F} 4 \geq 12.5 \mathrm{kPa})$. A fibrosis score equal to or greater than F2 was considered as significant fibrosis. ${ }^{23,24}$

\section{Statistical Analysis}

Statistical analyses were carried out using IBM SPSS Statistics for Windows (version 20.0 IBM Corp. Released 2011, Armonk, NY). Continuous data were assessed for normality using histograms as well as the skewness and kurtosis statistics, in which the values outside the range of -1 to +1 were considered as being non-normal. Demographic and clinical characteristics of participants in the study groups are presented as proportions, means \pm standard deviation, or medians with interquartile range (IQR) for skewed data. Between-group comparisons were performed using independent sample $t$ test and non-parametric Mann-Whitney U test for normally distributed and nonnormal data, respectively. Categorical data were compared using $\chi^{2}$ test if at least $80 \%$ of the cells had an expected count greater than 5, or Fisher exact test if this assumption was not met. A Spearman's partial correlation analysis was undertaken to examine the correlations of the fibrosis score with various clinical parameters, after controlling for age and gender. The significant predictors of hepatic fibrosis and steatosis by diabetes status were explored using binary logistic regression analyses and multivariable linear regression analyses with backward procedure, considering an alpha level of 0.157 for backward selection. In linear regression analyses, the candidate predictors for inclusion in the multivariable models in overall participants have been general covariates of age, gender, smoking status, diabetes status and statin medication as well as those with $P$ value $<0.1$ in the univariate correlation analysis with CAP score, including: BMI, waist-hip ratio (WHR), systolic blood pressure (SBP), fasting blood glucose (FBG), HOMA, TG, HDL, ALT, AST, GGT, A1C. For performing multivariable analyses in each study group, the candidate predictors consisted of gender and statin medication as well as those with $P$ value $<0.1$ in the univariate correlation analysis with CAP score in the study groups, including the BMI, WC/WHR, HOMA, TG, ALT, AST, GGT (a total of 9 variables). Similarly, the candidate variables for inclusion in logistic regression analysis in overall participants were general covariates of age, gender, smoking status and diabetes status as well as those with $P$ value $<0.1$ in the univariate correlation analysis with fibrosis score, including: BMI, WC, WHR, SBP, BPD, FBG, HOMA, TG, HDL, LDL, ALT, AST, GGT, A1C and CAP score. However, due to low effective sample size, we had limitations in performing logistic regression analyses in the study groups separately. Thus, only the results of the analysis in the overall participants have been presented. All tests were 2-tailed, and $P \leq 0.05$ was considered statistically significant.

\section{Results}

Of 200 participants enrolled initially, two participants without diabetes were excluded later because of positive $\mathrm{HBsAg}$ and anti-HCV test results. Accordingly, data from 100 individuals with T2DM, and 98 individuals without diabetes matched for age, sex, and BMI were used in the final analyses. Table 1 presents the demographic, clinical and biochemical characteristics of the participants by diabetes status. As expected, there was no significant difference between the two study groups in terms of age, sex, and BMI; however, those with diabetes had significantly higher mean SBP, diastolic blood pressure (DBP), fasting blood glucose, HbA1C, HOMA-IR, AST, GGT and total energy intake compared to their counterparts without diabetes (all $P$ values $<0.05$ ).

Results of the fibroscan test (Table 2) indicated that, despite comparable rates of NAFLD (S1-S3) in both study groups (T2DMs: $87 \%$ vs. non-T2DM: $78.6 \%, P=0.11$ ), individuals with diabetes had a significantly higher mean CAP score $(294.90 \pm 53.12$ vs. $269.78 \pm 45.05 \mathrm{~dB} / \mathrm{m} ; P$ $<0.001)$, resulting in a significantly higher rate of severe steatosis (S3) compared to those without diabetes $(50 \%$ vs. $31.6 \%, P=0.009)$. Similarly, our results showed a significantly higher mean fibrosis score in individuals with diabetes compared to that in individuals without diabetes [5.50 kPa (4.50-6.10) vs. $4.50 \mathrm{kPa}(4.00-5.50), P<0.001]$. Given that fibrosis levels greater than F2 are clinically more significant, affecting patient outcomes, individuals in this study were categorized as those with no or minimal fibrosis (F0-F1), and those with advanced fibrosis (F2F4). Advanced fibrosis was accordingly diagnosed in $13 \%$ of people with diabetes, while its frequency was $4 \%$ in individuals without diabetes $(P=0.02)$.

We also found that patients with diabetes and advanced fibrosis had significantly higher BMI, WC, WHR, ALT, AST, and CAP score compared to those with no or minimal fibrosis $(P<0.05)$; however, duration of diabetes did not affect fibrosis level in this study (Table 3 ). On the other hand, people without diabetes with and without advanced stages of liver fibrosis only differed significantly in BMI (Table 4).

Table 5 demonstrates the correlation of fibrosis scores with demographic and clinical parameters by diabetes status. BMI, WC, WHR, fasting insulin, GGT and CAP score had positive correlations with fibrosis scores in both groups. Also, HOMA-IR was correlated with hepatic fibrosis in patients with diabetes. However, the correlation did not remain statistically significant when they were adjusted for age, sex, BMI, and WC. 
Table 1. Characteristics of the Study Participants by Diabetes Status

\begin{tabular}{|c|c|c|c|}
\hline & People with Diabetes $(n=100)$ & People without Diabetes $(n=98)$ & $P$ Value \\
\hline Age (year) & $45.11 \pm 8.3$ & $44.93 \pm 8.5$ & 0.89 \\
\hline Female, n (\%) & $53(53 \%)$ & $52(53.1 \%)$ & 0.99 \\
\hline $\mathrm{BMI}\left(\mathrm{kg} / \mathrm{m}^{2}\right)$ & $30.00 \pm 4.79$ & $29.88 \pm 4.3$ & 0.85 \\
\hline WC (cm) & $102.68 \pm 12.01$ & $103.04 \pm 10.8$ & 0.82 \\
\hline WHR & $0.95 \pm 0.06$ & $0.94 \pm 0.05$ & 0.16 \\
\hline $\mathrm{SBP}(\mathrm{mm} \mathrm{Hg})$ & $123(114-131)$ & $115.5(108-124)$ & $<0.001$ \\
\hline $\mathrm{DBP}(\mathrm{mm} \mathrm{Hg})$ & $78(72-84)$ & $74(68-80)$ & 0.002 \\
\hline Glucose (mg/dL) & 136.50 (109.5-178.75) & $86(80-91)$ & $<0.001$ \\
\hline $\mathrm{HbA1C}(\%)$ & $8.00(6.50-9.30)$ & $5.40(5.20-5.60)$ & $<0.001$ \\
\hline HOMA-IR & $3.23(2.19-4.79)$ & $2.05(1.36-2.65)$ & $<0.001$ \\
\hline $\mathrm{TG}(\mathrm{mg} / \mathrm{dL})$ & $128.50(85.50-209.25)$ & $114.5(79.50-169.75)$ & 0.08 \\
\hline Total chol. (mg/dL) & $143.50(119.25-170.50)$ & $163.50(139.50-185.25)$ & 0.001 \\
\hline LDL-chol. (mg/dL) & $71.03 \pm 28.96$ & $92.54 \pm 25.36$ & $<0.001$ \\
\hline HDL-chol. (mg/dL) & $42.93 \pm 11.35$ & $43.82 \pm 10.20$ & 0.57 \\
\hline ALT (IU/L) & $16.50(12.00-22.75)$ & $18.50(13.75-26.25)$ & 0.03 \\
\hline AST (IU/L) & $20.00(17.00-28.00)$ & $17.00(15.00-23.00)$ & 0.005 \\
\hline GGT (IU/L) & $33.50(25.25-48.00)$ & $20.00(13.00-28.25)$ & $<0.001$ \\
\hline Total energy intake (kcal/d) & $2594.7(2235.2-3016.6)$ & $2376.1(1929.2-2935.7)$ & 0.02 \\
\hline Physical activity score ${ }^{b}$ (MET-min/wk) & $640.0(297.0-1626.0)$ & $879.0(370.5-2135.2)$ & 0.31 \\
\hline
\end{tabular}

Continuous variables are expressed as mean $\pm \mathrm{SD}$ or as median (IQR) for skewed data. Categorical variables are presented as $\mathrm{n}$ (\% within group). Betweengroup comparisons were performed using independent sample t-test and non-parametric Mann-Whitney $U$ test for normally distributed and skewed data, respectively. Categorical data were compared using $\chi 2$ test.

${ }^{\text {a }}$ Determined in 70 diabetic participants who did not take insulin medication; ${ }^{b}$ Evaluated in 99 diabetics and 98 non-diabetic participants.

Table 2. Hepatic Steatosis and Fibrosis Stratified by Diabetes Status

\begin{tabular}{|c|c|c|c|}
\hline & People with Diabetes $(n=100)$ & People without Diabetes $(n=98)$ & $P$ Value \\
\hline Fibrosis score $(\mathrm{kPa})$ & $5.50(4.50-6.10)$ & $4.50(4.00-5.50)$ & $<0.001$ \\
\hline Fibrosis stage, No. (\%) & & & 0.02 \\
\hline F0/F1 (No or minimal fibrosis) & 87 (87\%) & $94(95.9 \%)$ & \\
\hline F2-F4 (significant fibrosis) & $13(13 \%)$ & $4(4.1 \%)$ & \\
\hline CAP score $(\mathrm{dB} / \mathrm{m})^{\mathrm{a}, \mathrm{b}}$ & $294.48 \pm 3.92$ & $270.66 \pm 3.96$ & $<0.001$ \\
\hline Steatosis grade, No. (\%) & & & 0.009 \\
\hline SO-S2 & $50(50 \%)$ & $67(68.4)$ & \\
\hline S3 (sever steatosis) & $50(50 \%)$ & $31(31.6 \%)$ & \\
\hline
\end{tabular}

a Presented as estimated marginal means $\pm \mathrm{SE}$; Between-group comparisons were performed using ANCOVA and non-parametric Mann-Whitney $U$ test for normally distributed and skewed data, respectively. Categorical data were compared using $\chi^{2}$ test. ${ }^{b}$ Adjusted for age, sex, BMI.

Using multivariable linear regression analyses for the entire group (Table 6), diabetes status, BMI, WHR and ALT were significantly associated with higher CAP scores (model adjusted $\mathrm{R}^{2}: 0.47$ ), with BMI having the greatest influence $(\beta=0.49, P<0.001)$. A subsequent separate analysis for the two study groups highlighted BMI, WHR, AST and female gender as significant determinants of CAP scores in patients with diabetes (model adjusted $\mathrm{R}^{2}$ : 0.50 ); and WC, serum TG level, and statin medication in people without diabetes (model adjusted $\mathrm{R}^{2}$ : 0.43 ). Moreover, we found that in people with diabetes, BMI and WHR could each explain $43 \%$ and $18 \%$ of variations in CAP score, respectively.

Using binary logistic regression analyses, we revealed that AST (OR: 1.07, CI: 1.02-1.12, P: 0.01) and CAP score (OR: 1.03, CI: $1.02-1.05, P<0.001)$ were the main independent determinants of liver fibrosis in the entire population (Table 7 ).

\section{Discussion}

This study shows that people with diabetes are more likely to display more severe forms of steatosis and fibrosis compared to people without diabetes with the same age, sex and BMI. CAP score, HOMA-IR, higher BMI, higher WC, WHR, and AST were contributing factors to more severe NAFLD.

Parallel to the increased incidence of diabetes, NAFLD, as the most common cause of chronic liver diseases, has also increased. ${ }^{25,26}$ Recent studies showed NAFLD as a multisystem disease involving liver, cardiovascular, and 
Table 3. Demographic and Clinical Characteristics of the Participants with Diabetes by Liver Fibrosis Stage

\begin{tabular}{|c|c|c|c|}
\hline & \multicolumn{2}{|c|}{ Fibrosis Stage } & \multirow{2}{*}{$P$ Value between Group } \\
\hline & F0/F1 [n = $87(87 \%)]$ & F2-F4 [n = $13(13 \%]$ & \\
\hline Age (y) & $45.26 \pm 8.49$ & $44.07 \pm 7.77$ & 0.64 \\
\hline Female, No. (\%) & $49(56.3 \%)$ & $4(30.8 \%)$ & 0.14 \\
\hline $\mathrm{BMI}\left(\mathrm{kg} / \mathrm{m}^{2}\right)$ & $29.58 \pm 4.66$ & $32.85 \pm 4.87$ & 0.02 \\
\hline WC $(\mathrm{cm})$ & $101.15 \pm 11.38$ & $112.89 \pm 11.54$ & 0.001 \\
\hline $\mathrm{WC} / \mathrm{HC}$ & $0.95 \pm 0.06$ & $1.00 \pm 0.04$ & 0.003 \\
\hline $\mathrm{SBP}(\mathrm{mm} \mathrm{Hg})$ & $123.0(114.0-132.0)$ & $127.0(116.0-129.0)$ & 0.56 \\
\hline $\mathrm{DBP}(\mathrm{mm} \mathrm{Hg})$ & $77.0(72.0-84.0)$ & $78.0(71.5-81.5)$ & 0.92 \\
\hline $\mathrm{FBG}(\mathrm{mg} / \mathrm{dL})$ & 133 (109.0-181.0) & $148.0(116.0-156.0)$ & 0.82 \\
\hline $\mathrm{HbA1C}(\%)$ & $7.65(6.47-9.45)$ & $8.60(6.95-9.20)$ & 0.67 \\
\hline Fasting insulin $(\mu \mathrm{lU} / \mathrm{mL})$ & $11.30(7.80-14.70)$ & $20.90(12.5-34.30)$ & 0.005 \\
\hline HOMA-IR & $3.74(2.34-5.85)$ & $7.45(3.40-11.83)$ & 0.04 \\
\hline TG (mg/dL) & $130.0(88.0-216.0)$ & $99.0(81.0-180.0)$ & 0.30 \\
\hline Total chol. (mg/dL) & $147.0(121.0-173.0)$ & $123.0(106.5-148.5)$ & 0.04 \\
\hline LDL-chol. (mg/dL) & $72.95 \pm 29.89$ & $58.76 \pm 18.56$ & 0.10 \\
\hline HDL-chol. (mg/dL) & $43.31 \pm 11.60$ & $40.53 \pm 9.64$ & 0.42 \\
\hline ALT (IU/L) & $15.0(11.0-21.0)$ & $24.0(16.5-33.0)$ & 0.002 \\
\hline AST (IU/L) & $20.0(16.0-27.0)$ & $28.0(21.5-37.0)$ & 0.006 \\
\hline GGT (IU/L) & $32.0(25.0-47.0)$ & $45.0(31.0-57.0)$ & 0.09 \\
\hline Alk.ph (IU/L) & $66.0(53.0-84.0)$ & $79.0(55.0-89.0)$ & 0.36 \\
\hline Current smokers, No. (\%) & $26(29.9 \%)$ & $7(53.8 \%)$ & 0.12 \\
\hline Diabetes duration (y) & $7.0(5.0-11.0)$ & $7.0(3.5-10.0)$ & 0.39 \\
\hline \multicolumn{4}{|l|}{ Medication, No. (\%) } \\
\hline Insulin & $27(31.0 \%)$ & $3(23.1 \%)$ & 0.75 \\
\hline Oral glucose-lowering medication & $76(87.4 \%)$ & $12(92.3 \%)$ & 1.00 \\
\hline Statin & $49(56.3 \%)$ & $8(61.5 \%)$ & 0.77 \\
\hline CAP score $(\mathrm{dB} / \mathrm{m})$ & $286.75 \pm 51.12$ & $349.38 \pm 29.30$ & $<0.001$ \\
\hline Physical activity score (MET-min/wk) & $693.0(360.0-1638.0)$ & $393.0(24.7-969.7)$ & 0.08 \\
\hline
\end{tabular}

Continuous variables are expressed as mean \pm SD or as median (IQR) for skewed data unless otherwise noted. Categorical variables are presented as $\mathrm{n}$ (\% within liver fibrosis stage). Between-group comparisons were performed using independent sample t-test and non-parametric Mann-Whitney $U$ test for normally distributed and skewed data, respectively. Categorical data were compared using $\chi^{2}$ test.

cerebrovascular systems..$^{25,27}$ Diabetes and NAFLD increase the risk of overall mortality by 2.2 folds $^{28}$ and in people with diabetes, NAFLD increases the risk of cardiovascular events by 1.87 folds. ${ }^{29}$ In addition, diabetes increases the risk of hepatocellular carcinoma as well as hepatocellular carcinoma mortality by $2-3$ folds. ${ }^{30-32}$ Therefore, timely detection and treatment of liver steatosis and fibrosis is crucial to improve long-term prognosis of individuals with diabetes. However, up to now, routine screening has not been recommended for NAFLD due to cost, limited treatment options, and current ineffectiveness of diagnostic modalities. ${ }^{33}$ Furthermore, there is controversy regarding whether a screening program should be implemented for the total population or only high risk groups, namely people with diabetes. ${ }^{34}$

Although liver biopsy is the gold standard to assess NAFLD, it is an invasive method with a fatality rate of $0.05 \% .{ }^{35}$ Moreover, it is not feasible in a large population. Therefore, only a limited number of studies have used liver biopsy to assess NAFLD in patients with diabetes. ${ }^{7,36,37}$ Some studies used magnetic resonance spectroscopy
(MRS) to evaluate NAFLD, ${ }^{38,39}$ though it is expensive with limited accessibility. Considering MRS > 5.5\% as indicator of NAFLD, $60.3 \%$ of patients with diabetes were diagnosed with NAFLD in one study. ${ }^{39}$

In another study on 100 patients with diabetes conducted by Doycheva et al, the prevalence of advanced fibrosis (magnetic resonance elastography [MRE] > $3.6 \mathrm{kPa}$ ) was $7.1 \%$ using MRE. ${ }^{28}$ In a study that was conducted by Park et al, ${ }^{40}$ MRE was compared with TE with XL probe in detection of fibrosis and steatosis in patients with biopsy-proven NAFLD. They found MRI to have higher diagnostic accuracy for fibrosis and steatosis in NAFLD patients, ${ }^{41-43}$ while TE had higher negative predictive value for diagnosing significant fibrosis (stages 2-4), severe fibrosis (stages 3-4), as well as cirrhosis. ${ }^{44,45}$ Furthermore, in another study conducted by Eddowes et al, it was demonstrated that TE has high applicability as well as a low failure rate in patients with potential NAFLD with area under the receiver operating characteristic curve values ranging from 0.7 to 0.89 , showing that histological components or choice of probe do not influence the 
Table 4. Demographic and Clinical Characteristics of the Participants without Diabetes by Liver Fibrosis Stage

\begin{tabular}{|c|c|c|c|}
\hline & \multicolumn{2}{|c|}{ Fibrosis Stage } & \multirow{2}{*}{$\begin{array}{c}P \text { Value between } \\
\text { Group }\end{array}$} \\
\hline & F0/F1 [n = $94(95.9 \%)]$ & F2-F4 $[n=4(4.01 \%)]$ & \\
\hline Age $(y)$ & $44.92 \pm 8.66$ & $45.25 \pm 7.63$ & 0.94 \\
\hline Female, $n(\%)$ & $51(54.3 \%)$ & $1(25 \%)$ & 0.34 \\
\hline BMI $\left(\mathrm{kg} / \mathrm{m}^{2}\right)$ & $29.70 \pm 4.26$ & $34.12 \pm 4.37$ & $<0.05$ \\
\hline WC $(\mathrm{cm})$ & $102.64 \pm 10.64$ & $112.45 \pm 12.21$ & 0.08 \\
\hline $\mathrm{WC} / \mathrm{HC}$ & $0.94 \pm 0.05$ & $0.097 \pm 0.06$ & 0.24 \\
\hline $\mathrm{SBP}(\mathrm{mm} \mathrm{Hg})$ & 115.5 (108.0-124.0) & $117.5(87.2-126.0)$ & 0.93 \\
\hline $\mathrm{DBP}(\mathrm{mm} \mathrm{Hg})$ & $73.5(68.0-80.0)$ & $75.0(71.2-75.7)$ & 0.80 \\
\hline $\mathrm{FBG}(\mathrm{mg} / \mathrm{dL})$ & $86.0(79.0-91.0)$ & $88.5(85.0-91.25)$ & 0.48 \\
\hline $\mathrm{HbA1C}(\%)$ & $5.4(5.20-5.60)$ & $5.65(5.15-6.00)$ & 0.22 \\
\hline Fasting insulin $(\mu \mathrm{lU} / \mathrm{mL})$ & $9.24(6.73-12.66)$ & $18.58(7.23-22.84)$ & 0.16 \\
\hline HOMA-IR & $2.03(1.36-2.62)$ & $4.14(1.53-5.05)$ & 0.14 \\
\hline $\mathrm{TG}(\mathrm{mg} / \mathrm{dL})$ & $113.5(79.5-172.5)$ & $127.5(60.0-162.7)$ & 0.86 \\
\hline Total chol. (mg/dL) & $163.5(140.0-185.0)$ & $152.0(123.7-204.2)$ & 0.76 \\
\hline LDL-C (mg/dL) & $92.54 \pm 24.67$ & $92.50 \pm 43.79$ & 1.00 \\
\hline $\mathrm{HDL}-\mathrm{C}(\mathrm{mg} / \mathrm{dL})$ & $43.80 \pm 9.87$ & $44.25 \pm 18.55$ & 0.93 \\
\hline ALT (IU/L) & $18.00(13.00-26.00)$ & $35.00(17.25-76.75)$ & 0.11 \\
\hline AST (IU/L) & $17.00(15.00-23.00)$ & $31.00(17.50-44.50)$ & 0.07 \\
\hline GGT (IU/L) & $20.00(13.00-28.00)$ & $34.00(11.50-58.00)$ & 0.34 \\
\hline ALP (IU/L) & $65.00(53.75-83.25)$ & $72.00(61.25-90.25)$ & 0.36 \\
\hline Current smokers, $n(\%)$ & $19(20.2 \%)$ & $0(0 \%)$ & 1.00 \\
\hline \multicolumn{4}{|l|}{ Medication, $n(\%)$} \\
\hline Statin & $11(11.7 \%)$ & $1(25 \%)$ & 0.41 \\
\hline Fibrosis score (kPa) & $4.50(4.00-5.50)$ & $9.50(9.12-12.12)$ & $<0.001$ \\
\hline CAP score $(\mathrm{dB} / \mathrm{m})$ & $268.02 \pm 44.85$ & $311.25 \pm 29.54$ & 0.06 \\
\hline Physical activity score (MET-min/wk) & $879.0(370.50-2165.25)$ & $748.5(104.25-1161.0)$ & 0.44 \\
\hline
\end{tabular}

Continuous variables are expressed as mean \pm SD or as median (IQR) for skewed data unless otherwise noted. Categorical variables are presented as $\mathrm{n}(\%$ within liver fibrosis stage). Between-group comparisons were performed using independent sample t-test and Mann-Whitney $U$ test for normally distributed and skewed data, respectively. Categorical data were compared using Fisher exact test.

Table 5. Correlation of Fibrosis Score with Different Demographic and Clinical Parameters by Diabetes Status*

\begin{tabular}{|c|c|c|c|c|c|c|}
\hline & \multicolumn{2}{|c|}{ People with Diabetes } & \multicolumn{2}{|c|}{ People without Diabetes } & \multicolumn{2}{|l|}{ Overall } \\
\hline & Correlation Coefficient $^{a}$ & $P$ Value & Correlation Coefficient $^{a}$ & $P$ Value & Correlation Coefficient ${ }^{\mathrm{a}}$ & $P$ Value \\
\hline Age $(y)^{b}$ & 0.01 & 0.92 & -0.09 & 0.41 & -0.01 & 0.85 \\
\hline BMI $\left(\mathrm{kg} / \mathrm{m}^{2}\right)$ & 0.35 & $<0.001$ & 0.47 & $<0.001$ & 0.41 & $<0.001$ \\
\hline WC $(\mathrm{cm})$ & 0.40 & $<0.001$ & 0.42 & $<0.001$ & 0.40 & $<0.001$ \\
\hline WHR & 0.32 & 0.001 & 0.30 & 0.003 & 0.33 & $<0.001$ \\
\hline $\mathrm{SBP}(\mathrm{mmHg})^{c}$ & 0.25 & 0.02 & -0.10 & 0.36 & 0.12 & 0.03 \\
\hline $\mathrm{DBP}(\mathrm{mmHg})^{\mathrm{c}}$ & 0.26 & 0.01 & -0.05 & 0.66 & 0.15 & $<0.05$ \\
\hline $\mathrm{FBG}(\mathrm{mg} / \mathrm{dL})^{\mathrm{c}}$ & 0.002 & 0.99 & -0.09 & 0.37 & 0.22 & 0.002 \\
\hline $\mathrm{HbA} 1 \mathrm{C}(\%)^{c}$ & 0.03 & 0.77 & 0.16 & 0.12 & 0.31 & $<0.001$ \\
\hline Insulin $(\mu \mathrm{IU} / \mathrm{mL})^{\mathrm{c,d}}$ & 0.38 & 0.001 & 0.31 & 0.003 & 0.33 & $<0.001$ \\
\hline HOMA-IR ${ }^{c, d}$ & 0.23 & 0.07 & 0.28 & 0.006 & 0.35 & $<0.001$ \\
\hline $\mathrm{TG}(\mathrm{mg} / \mathrm{dL})^{\mathrm{c}}$ & -0.02 & 0.85 & 0.14 & 0.17 & 0.11 & 0.15 \\
\hline Total chol $(\mathrm{mg} / \mathrm{dL})^{\mathrm{c}}$ & -0.12 & 0.26 & 0.05 & 0.67 & -0.12 & 0.11 \\
\hline LDL-C $(\mathrm{mg} / \mathrm{dL})^{\mathrm{c}}$ & -0.13 & 0.23 & -0.04 & 0.72 & -0.20 & 0.006 \\
\hline $\mathrm{HDL}-\mathrm{C}(\mathrm{mg} / \mathrm{dL})^{\mathrm{c}}$ & -0.08 & 0.43 & -0.11 & 0.29 & -0.15 & 0.04 \\
\hline ALT (IU/L) & 0.46 & $<0.001$ & 0.27 & 0.008 & 0.28 & $<0.001$ \\
\hline AST (IU/L) & 0.35 & $<0.001$ & 0.18 & 0.08 & 0.31 & $<0.001$ \\
\hline GGT (IU/L) & 0.36 & $<0.001$ & 0.31 & 0.003 & 0.41 & $<0.001$ \\
\hline Diabetes duration (y) & 0.07 & 0.49 & - & - & - & - \\
\hline CAP score $(\mathrm{dB} / \mathrm{m})$ & 0.43 & $<0.001$ & 0.47 & $<0.001$ & 0.49 & $<0.001$ \\
\hline Physical activity score (MET-min/wk) & -0.22 & 0.03 & 0.001 & 0.99 & -0.11 & 0.12 \\
\hline
\end{tabular}

Correlations between variables of interest were assessed using a Spearman's partial correlation analyses.

* Controlled for age and sex unless otherwise noted; ${ }^{a}$ Spearman rank correlation coefficient. ${ }^{b}$ Controlled for sex; ${ }^{\mathrm{c}}$ Controlled for age, sex, BMI, WC. ${ }^{\mathrm{d}}$ Determined in 70 diabetic participants who did not take insulin medication. 
Table 6. Results of the Linear Regression Models Evaluating the Association of CAP Score with Different Clinical Parameters in Overall and in Each Study Group, Separately

\begin{tabular}{|c|c|c|c|c|}
\hline & Independent Variables & Unstandardized Coefficients (B) & $95 \% \mathrm{Cl}$ for $\mathrm{B}$ & $P$ Value \\
\hline \multirow{5}{*}{ Overall } & Diabetes status & -24.82 & $-35.74,-13.90$ & $<0.001$ \\
\hline & WHR & 195.12 & $84.10,306.15$ & 0.001 \\
\hline & BMI $\left(\mathrm{kg} / \mathrm{m}^{2}\right)$ & 5.14 & $3.81,6.47$ & $<0.001$ \\
\hline & ALT (IU/L) & 0.67 & $0.22,1.11$ & 0.003 \\
\hline & Gender & -12.20 & $-25.30,0.90$ & 0.068 \\
\hline \multirow{4}{*}{ People with diabetes } & Gender & -23.10 & $-41.13,-5.07$ & 0.01 \\
\hline & WHR & 262.68 & $119.51,405.85$ & $<0.001$ \\
\hline & $\mathrm{BMI}\left(\mathrm{kg} / \mathrm{m}^{2}\right)$ & 6.01 & $4.33,7.70$ & $<0.001$ \\
\hline & AST (IU/L) & 0.78 & $0.05,1.52$ & 0.04 \\
\hline \multirow{4}{*}{ People without diabetes } & WC $(\mathrm{cm})$ & 2.21 & $1.53,2.89$ & $<0.001$ \\
\hline & TG (mg/dL) & 0.09 & $-0.001,0.18$ & 0.05 \\
\hline & Statin Med. & 32.20 & $11.31,53.09$ & 0.003 \\
\hline & ALT (IU/L) & 0.39 & $-0.08,0.87$ & 0.10 \\
\hline
\end{tabular}

Dependent variable: CAP score; Overall: $\mathrm{R}^{2}=0.48$ (adjusted $\mathrm{R}^{2}=0.47$ ); People with Diabetes: $\mathrm{R}^{2}=0.53$ (adjusted $\mathrm{R}^{2}=0.50$ ); People without Diabetes: $\mathrm{R}^{2}=0.46$ (adjusted $\mathrm{R}^{2}=0.43$ ).

\section{measurements. ${ }^{46}$}

Goh et $\mathrm{al}^{15}$ reported different clinical spectra of NAFLD in people with diabetes compared to people without diabetes using liver biopsy as the gold standard. The mean BMI, AST, and ALT in people with diabetes in that study were higher compared to our population with diabetes. Consistent with our findings, duration of diabetes was not different between people with and without significant fibrosis. However, advanced fibrosis was reported to be present in $40.3 \%$ of people with diabetes compared to $17 \%$ in those without diabetes. In our study, significant fibrosis was found in $13 \%$ of people with diabetes, and $4 \%$ of those without diabetes. This may be due to different stages of chronic inflammation, ${ }^{47}$ oxidative stress, ${ }^{48}$ as well as the up-regulation of hepatotoxic inflammatory cytokines in diabetes. ${ }^{49}$ Other mechanisms might be highlevel of glucose and insulin concentrations which can stimulate connective tissue growth factor expression - a determining factor in progressive hepatic fibrosis. ${ }^{49}$ In a study conducted by Nakahara et $\mathrm{al}^{50}$ in a large patient cohort $(\mathrm{N}=1365)$ with biopsy-proven NAFLD, they found a parallel rise in the prevalence of diabetes and degree of fibrosis, indicating diabetes as a significant risk factor for advanced fibrosis.

Although the prevalence of advanced fibrosis may be lower among people without diabetes, due to larger population of people without diabetes, the absolute number of severe NAFLD in individuals without diabetes may not be lower than people with diabetes.

In another study on 939 patients with T2DM in Edinburgh, the prevalence of NAFLD was $42.6 \%$ using ultrasound. ${ }^{6}$ The disparity in the prevalence of NAFLD between different studies may be due to the variations in sensitivity of diagnostic modalities as well as different methods of sample selection, dietary, lifestyle habits of participants, and different patient characteristics. ${ }^{51}$

The results of our study showed that the female gender was a risk factor for NAFLD in patients with diabetes. While in another cross-sectional study on 2839 patients with diabetes, the prevalence of NAFLD was reported to be $71.1 \%$ in men and $68 \%$ in women, though the age range was not different to ours. But the duration of diabetes in that study was higher in people with NAFLD compared to ours. ${ }^{29}$ Consistent with our findings, in a study conducted by Summart et $\mathrm{al}^{52}$ on 34709 participants (27073 females and 7636 males), they found a higher NAFLD prevalence in women, which increased with age, with the largest difference among the population of 56-60 years old as well as patients with diabetes. The age range can be justified by natural physiologic changes in women during the postmenopausal period including insulin resistance, central obesity, adipose distribution,

Table 7. The Result of Multivariable Logistic Regression Analysis Evaluating the Association of Clinical Parameters with the Presence of Significant Fibrosis in Overall Participants

\begin{tabular}{|c|c|c|c|c|}
\hline \multirow{2}{*}{ Variables } & \multirow{2}{*}{$P$ Value } & \multirow{2}{*}{ Odds Ratio } & \multicolumn{2}{|c|}{$95 \% \mathrm{Cl}$ for $\mathrm{OR}$} \\
\hline & & & Lower & Upper \\
\hline AST (IU/L) & 0.01 & 1.07 & 1.02 & 1.12 \\
\hline Cap score $(\mathrm{dB} / \mathrm{m})$ & $<0.001$ & 1.03 & 1.02 & 1.05 \\
\hline HOMA-IR & 0.02 & 1.10 & 1.01 & 1.19 \\
\hline TG (mg/dL) & 0.01 & 0.98 & 0.97 & 0.997 \\
\hline $\mathrm{SBP}(\mathrm{mm} \mathrm{Hg})$ & 0.07 & 0.95 & 0.89 & 1.004 \\
\hline Gender & 0.08 & 3.56 & 0.88 & 14.51 \\
\hline LDL (mg/dL) & 0.11 & 0.98 & 0.96 & 1.004 \\
\hline
\end{tabular}

Dependent variable: No or minimal fibrosis (F0-F1)/ significant fibrosis (F2-F4); SBP, Systolic blood pressure. 
and sex hormones. ${ }^{53}$ Eliminating the effect of estrogen as a powerful antioxidant may lead to fatty acid oxidant decrease, while increasing lipogenesis within the liver resulting in visceral fat accumulation and NAFLD. ${ }^{54}$

In addition, estrogen is a protective factor against diabetes $^{55}$; therefore, the risk of NAFLD increases in the postmenopausal period. Moreover, insulin resistance in multiple organs such as the liver, muscle and adipose tissue is accompanied by higher gluconeogenesis in the liver, insulin clearance reduction, and impaired glucose uptake by muscles, all of which lead to elevated plasma glucose concentrations. ${ }^{56}$ Insulin resistance in the adipose tissue can also result in higher levels of free fatty acids and inflammatory cytokines. ${ }^{14}$ Therefore, transaminase levels increase in patients with NAFLD; however, it may not occur in all patients with diabetes. ${ }^{57}$

Moreover, Our study demonstrated that BMI, WC, WHR, HOMA-IR, and CAP score were associated with advanced liver fibrosis, while in a study conducted by Zhao et $\mathrm{al},{ }^{58}$ older age, high BMI, smoking, and low PLT levels were reported as independent risk factors for liver fibrosis in patients with diabetes.

Consistent with the results of the study conducted by Zhao et al, our study revealed that high blood pressure as well as GGT and HOMA-IR had a significant association with hepatic steatosis in patients with diabetes. In addition, HOMA-IR has been reported as an independent risk factor for NAFLD and liver fibrosis both in people with diabetes ${ }^{59}$ and without diabetes. ${ }^{4}$ Therefore, it is reasonable to recommend hepatic steatosis screening in patients with diabetes, especially those who are overweight with high GGT and HOMA-IR as well as high blood pressure.

GGT also had a significant association with hepatic steatosis in individuals without diabetes. GGT, as one of the key enzymes responsible for glutathione metabolism, has been shown to be closely related with cardiovascular disease as well as central obesity. ${ }^{60}$ Our study also demonstrated higher GGT in grade 3 steatosis in patients with diabetes compared to individuals without diabetes with grade 3 steatosis, suggesting GGT as a biomarker for hepatic steatosis in patients with diabetes. ${ }^{58}$

\section{Strength and Limitation}

To date, a limited number of studies have explored liver steatosis and fibrosis in people with T2DM compared to individuals without diabetes matched for age, sex, and BMI. The strengths of our study include recruitment of subjects from diabetes clinics in routine clinical practice and age, sex and BMI matched individuals without diabetes, pragmatic use of a combination of readily available biomarkers, and use of transient elastography for liver assessments. In addition, diet and physical activity were assessed. On the other hand, our study had some limitations. The platelet count, as one possible risk factor for liver fibrosis, was not measured. In addition, the sample size was relatively small and patients were enrolled in a single center. A multicenter controlled randomized study is warranted to validate the findings of our study.

In conclusion, individuals with diabetes are more likely to have severe fibrosis. Obesity, central obesity, female gender, elevated liver enzymes, and higher degree of insulin resistance are risk factors predicting severity of NAFLD.

The findings highlight the potential importance of early screening for NAFLD in high risk subgroups of patients with diabetes as well as overweight people with high GGT, high HOMA-IR and high blood pressure to initiate timely behavioral and/or medical intervention.

\section{Authors' Contribution}

Study concept and design: SN, MEKh, and MM; statistical analysis: FASh and MEKh; analysis and interpretation of data: FASh, RA, $\mathrm{HP}, \mathrm{SN}$, and MEKh; drafting of the manuscript: RA, HP, and FASh; critical revision of the manuscript for important intellectual content: HP, FASh, RA, SN, MM, and MEKh.

\section{Conflict of Interest Disclosures}

Nothing to declare.

\section{Ethical Statement}

The Iran University of Medical Sciences Ethics committee approved the study protocol. The study procedures were carried out in accordance with the principles of the Declaration of Helsinki. Informed consent was obtained from all participants.

\section{Acknowledgements}

The authors wish to thank to all the patients who participated in this study.

\section{References}

1. Ding W, Fan J, Qin J. Association between nonalcoholic fatty liver disease and colorectal adenoma: a systematic review and meta-analysis. Int J Clin Exp Med. 2015;8 (1):322-33.

2. Moghaddasifar I, Lankarani K, Moosazadeh M, Afshari M, Ghaemi A, Aliramezany M, et al. Prevalence of non-alcoholic fatty liver disease and its related factors in Iran. Int J Organ Transplant Med. 2016;7(3):149-60.

3. Kitade $\mathrm{H}$, Chen $\mathrm{G}, \mathrm{Ni} \mathrm{Y}, \mathrm{Ota} \mathrm{T}$. Nonalcoholic fatty liver disease and insulin resistance: New insights and potential new treatments. Nutrients. 2017;9(4):387. doi: 10.3390/ nu9040387.

4. Fujii $\mathrm{H}$, Imajo $\mathrm{K}$, Yoneda $\mathrm{M}$, Nakahara $\mathrm{T}$, Hyogo $\mathrm{H}$, Takahashi $\mathrm{H}$, et al. HOMA-IR: An independent predictor of advanced liver fibrosis in nondiabetic non-alcoholic fatty liver disease. J Gastroenterol Hepatol. 2019;34(8):1390-5. doi: 10.1111/ jgh.14595.

5. Ramachandran TM, Rajneesh AHR, Zacharia GS, Adarsh RP. Cirrhosis of liver and diabetes mellitus: The diabolic duo? J Clin Diagn Res. 2017;11(9):OC01-OC05. doi: 10.7860/ JCDR/2017/30705.10529.

6. Chalasani N, Younossi Z, Lavine JE, Diehl AM, Brunt EM, Cusi $\mathrm{K}$, et al. The diagnosis and management of non-alcoholic fatty liver disease: Practice guideline by the American association for the study of liver diseases, American college of gastroenterology, and the American Gastroenterological Association. Hepatology. 2012;55(6):2005-23. doi: 10.1002/ hep. 25762.

7. Bohte $A E$, van Werven JR, Bipat $S$, Stoker J. The diagnostic accuracy of US, CT, MRI and 1H-MRS for the evaluation of hepatic steatosis compared with liver biopsy: a meta-analysis. Eur Radiol. 2011;21(1):87-97. doi: 10.1007/s00330-010- 
1905-5.

8. Yoneda M, Suzuki K, Kato S, Fujita K, Nozaki Y, Hosono K, et al. Nonalcoholic fatty liver disease: Us-based acoustic radiation force impulse elastography. Radiology. 2010;256(2):640-7. doi: 10.1148/radiol.10091662

9. Kim BH, Lee JM, Lee YJ, Lee KB, Suh KS, Han JK, et al. Mr elastography for noninvasive assessment of hepatic fibrosis: Experience from a tertiary center in Asia. J Magn Reson Imaging. 2011;34(5):1110-6. doi: 10.1002/jmri.22723.

10. Chen J, Talwalkar JA, Yin M, Glaser KJ, Sanderson SO, Ehman RL. Early detection of nonalcoholic steatohepatitis in patients with nonalcoholic fatty liver disease by using MR elastography. Radiology. 2011;259(3):749-56. doi: 10.1148/ radiol.11101942.

11. Kim HJ, Lee HK, Cho JH, Yang HJ. Quantitative comparison of transient elastography(TE), shear wave elastography(SWE) and liver biopsy results of patients with chronic liver disease. J Phys Ther Sci. 2015;27(8):2465-8. doi: 10.1589/jpts.27.2465.

12. Foucher J, Chanteloup E, Vergniol J, Castera L, Le Bail B, Adhoute X, et al. Diagnosis of cirrhosis by transient elastography (fibroscan): A prospective study. Gut. 2006;55(3):403-8. doi: 10.1136/gut.2005.069153.

13. de Lédinghen $\mathrm{V}$, Hiriart J-B, Vergniol J, Merrouche W, Bedossa $\mathrm{P}$, Paradis V. Controlled attenuation parameter (CAP) with the $\mathrm{XL}$ probe of the Fibroscan $\AA$ : a comparative study with the $\mathrm{m}$ probe and liver biopsy. Dig Dis Sci. 2017;62(9):2569-2577. doi: 10.1007/s10620-017-4638-3.

14. Rich NE, Oji S, Mufti AR, Browning JD, Parikh ND, Odewole $M$, et al. Racial and ethnic disparities in nonalcoholic fatty liver disease prevalence, severity, and outcomes in the united states: a systematic review and meta-analysis. Clin Gastroenterol Hepatol. 2018;16(2):198-210.e2. doi: 10.1016/j.cgh.2017.09.041.

15. Goh GB-B, Pagadala MR, Dasarathy J, Unalp-Arida A, Sargent $R$, Hawkins $C$, et al. Clinical spectrum of non-alcoholic fatty liver disease in diabetic and non-diabetic patients. BBA Clin. 2014;3:141-5. doi: 10.1016/j.bbacli.2014.09.001.

16. Harrell FE. Regression modeling strategies: With applications to linear models, logistic regression, and survival analysis. New York, USA: Springer-Verlag; 2001.

17. Peduzzi P, Concato J, Kemper E, Holford TR, Feinstein AR. A simulation study of the number of events per variable in logistic regression analysis. J Clin Epidemiol. 1996;49(12):1373-9. doi: 10.1016/s0895-4356(96)00236-3.

18. Matthews D, Hosker J, Rudenski A, Naylor B, Treacher D, Turner R. Homeostasis model assessment: Insulin resistance and $\beta$-cell function from fasting plasma glucose and insulin concentrations in man. Diabetologia. 1985;28(7):412-9. doi: 10.1007/BF00280883.

19. Friedewald WT, Levy RI, Fredrickson DS. Estimation of the concentration of low-density lipoprotein cholesterol in plasma, without use of the preparative ultracentrifuge. Clin Chem. 1972;18(6):499-502.

20. International physical activity questionnaires IPAQ: Short last 7 days self-administered format. 2002. Available from: https:// evaluationframework.sportengland.org/media/1084/2015 ipaqsf.pdf. Accessed 2018 Jan 30.

21. Sasso M, Beaugrand $M$, de Ledinghen $V$, Douvin C, Marcellin $\mathrm{P}$, Poupon R, et al. Controlled attenuation parameter (CAP): a novel $\mathrm{VCTE}^{\mathrm{TM}}$ guided ultrasonic attenuation measurement for the evaluation of hepatic steatosis: preliminary study and validation in a cohort of patients with chronic liver disease from various causes. Ultrasound Med Biol. 2010;36(11):182535. doi: 10.1016/j.ultrasmedbio.2010.07.005

22. Yen $\mathrm{YH}$, Chen JB, Cheng BC, Chen JF, Chang KC, Tseng PL, et al. Using controlled attenuation parameter combined with ultrasound to survey non-alcoholic fatty liver disease in hemodialysis patients: A prospective cohort study. PLoS One. 2017;12(4):e0176027. doi: 10.1371/journal.pone.0176027. 23. Myers RP, Elkashab M, Ma M, Crotty P, Pomier-Layrargues G. Transient elastography for the noninvasive assessment of liver fibrosis: A multicentre Canadian study. Can J Gastroenterol. 2010;24(11):661-70. doi: 10.1155/2010/153986.

24. Kim MK, Kim JH, Park K, Lee SB, Nam JS, Kang S, et al. Relationship between the triglyceride glucose index and the presence and fibrosis of nonalcoholic fatty liver disease in Korean adults. Diabetes. 2018;67(Suppl 1):612-P. doi: 10.2337/db18-612-P.

25. European Association for the Study of the Liver (EASL); European Association for the Study of Diabetes (EASD); European Association for the Study of Obesity (EASO). EASL-EASD-EASO Clinical Practice Guidelines for the Management of Non-Alcoholic Fatty Liver Disease. Obes Facts. 2016;9(2):65-90. doi: 10.1159/000443344.

26. White DL, Thrift AP, Kanwal F, Davila J, El-Serag HB. Incidence of hepatocellular carcinoma in all 50 United States, from 2000 through 2012. gastroenterology. 2017;152(4):812-820.e5. doi: 10.1053/j.gastro.2016.11.020.

27. Lonardo A, Sookoian S, Pirola CJ, Targher G. Non-alcoholic fatty liver disease and risk of cardiovascular disease. Metabolism. 2016;65(8):1136-50. doi: 10.1016/j.metabol.2015.09.017.

28. Doycheva I, Cui J, Nguyen P, Costa EA, Hooker J, Hofflich $\mathrm{H}$, et al. Non-invasive screening of diabetics in primary care for NAFLD and advanced fibrosis by MRI and MRE. Aliment Pharmacol Ther. 2016;43(1):83-95. doi: 10.1111/apt.13405.

29. Targher G, Bertolini L, Rodella S, Tessari R, Zenari L, Lippi G, et al. Nonalcoholic fatty liver disease is independently associated with an increased incidence of cardiovascular events in type 2 diabetic patients. Diabetes Care. 2007;30(8):2119-21. doi: 10.2337/dc07-0349.

30. Davila J, Morgan R, Shaib Y, McGlynn K, El-Serag H. Diabetes increases the risk of hepatocellular carcinoma in the united states: A population based case control study. Gut. 2005;54(4):533-9. doi: 10.1136/gut.2004.052167.

31. Wang P, Kang D, Cao W, Wang Y, Liu Z. Diabetes mellitus and risk of hepatocellular carcinoma: A systematic review and meta-analysis. Diabetes Metab Res Rev. 2012;28(2):109-22. doi: 10.1002/dmrr.1291.

32. Chiang $\mathrm{CH}$, Lee LT, Hung $\mathrm{SH}$, Lin WY, Hung HF, Yang WS, et al. Opposite association between diabetes, dyslipidemia, and hepatocellular carcinoma mortality in the middle-aged and elderly. Hepatology. 2014;59(6):2207-15. doi: 10.1002/ hep. 27014.

33. Zetterman RK. NAFLD is a growing problem. Medscape May 18, 2018. Available from: https://www.medscape.com/ viewarticle/896577.

34. Targher G, Day CP, Bonora E. Risk of cardiovascular disease in patients with nonalcoholic fatty liver disease. N Engl J Med. 2010;363(14):1341-50. doi: 10.1056/NEJMra0912063.

35. Meisamy S, Hines CD, Hamilton G, Sirlin CB, McKenzie CA, Yu H, et al. Quantification of hepatic steatosis with T1independent, T2-corrected MR imaging with spectral modeling of fat: blinded comparison with MR spectroscopy. Radiology. 2011;258(3):767-75. doi: 10.1148/radiol.10100708.

36. Lazo M, Hernaez R, Eberhardt MS, Bonekamp S, Kamel I, Guallar E, et al. Prevalence of nonalcoholic fatty liver disease in the united states: The third national health and nutrition examination survey, 1988-1994. Am J Epidemiol. 2013;178(1):38-45. doi: 10.1093/aje/kws448.

37. Pais R, Charlotte F, Fedchuk L, Bedossa P, Lebray P, Poynard $\mathrm{T}$, et al. A systematic review of follow-up biopsies reveals disease progression in patients with non-alcoholic fatty liver. J Hepatol. 2013;59(3):550-6. doi: 10.1016/j.jhep.2013.04.027.

38. Yokoo T, Bydder M, Hamilton G, Middleton MS, Gamst AC, Wolfson $T$, et al. Nonalcoholic fatty liver disease: diagnostic and fat-grading accuracy of low-flip-angle multiecho gradient- 
recalled-echo MR imaging at 1.5 T. Radiology. 2009;251(1):6776. doi: 10.1148/radiol.2511080666.

39. Yokoo T, Shiehmorteza M, Hamilton G, Wolfson T, Schroeder ME, Middleton MS, et al. Estimation of hepatic protondensity fat fraction by using MR imaging at 3.0 T. Radiology. 2011;258(3):749-59. doi: 10.1148/radiol.10100659.

40. Park CC, Nguyen P, Hernandez C, Bettencourt R, Ramirez $\mathrm{K}$, Fortney $\mathrm{L}$, et al. Magnetic resonance elastography vs transient elastography in detection of fibrosis and noninvasive measurement of steatosis in patients with biopsyproven nonalcoholic fatty liver disease. Gastroenterology. 2017;152(3):598-607.e2. doi: 10.1053/j.gastro.2016.10.026.

41. Loomba R, Wolfson T, Ang B, Hooker J, Behling C, Peterson $M$, et al. Magnetic resonance elastography predicts advanced fibrosis in patients with nonalcoholic fatty liver disease: A prospective study. Hepatology. 2014;60(6):1920-8. doi: 10.1002/hep.27362.

42. Permutt Z, Le TA, Peterson MR, Seki E, Brenner DA, Sirlin C, et al. Correlation between liver histology and novel magnetic resonance imaging in adult patients with non-alcoholic fatty liver disease - MRI accurately quantifies hepatic steatosis in NAFLD. Aliment Pharmacol Ther. 2012;36(1):22-9. doi: 10.1111/j.1365-2036.2012.05121.x.

43. Tang A, Desai A, Hamilton G, Wolfson T, Gamst A, Lam J, et al. Accuracy of MR imaging-estimated proton density fat fraction for classification of dichotomized histologic steatosis grades in nonalcoholic fatty liver disease. Radiology. 2015;274(2):41625. doi: 10.1148/radiol.14140754.

44. Pavlov CS, Casazza G, Nikolova D, Tsochatzis E, Burroughs AK, Ivashkin VT, et al. Transient elastography for diagnosis of stages of hepatic fibrosis and cirrhosis in people with alcoholic liver disease. Cochrane Database Syst Rev. 2015;1(1):CD010542. doi: 10.1002/14651858.CD010542.pub2.

45. Wong VWS, Vergniol J, Wong GLH, Foucher J, Chan HLY, Le Bail B, et al. Diagnosis of fibrosis and cirrhosis using liver stiffness measurement in nonalcoholic fatty liver disease. Hepatology. 2010;51(2):454-62. doi: 10.1002/hep.23312.

46. Eddowes PJ, Sasso M, Allison M, Tsochatzis E, Anstee QM, Sheridan D, et al. Accuracy of fibroscan controlled attenuation parameter and liver stiffness measurement in assessing steatosis and fibrosis in patients with nonalcoholic fatty liver disease. Gastroenterology. 2019;156(6):1717-30. doi: 10.1053/j.gastro.2019.01.042..

47. Davì G, Guagnano MT, Ciabattoni G, Basili S, Falco A, Marinopiccoli $M$, et al. Platelet activation in obese women: Role of inflammation and oxidant stress. JAMA. 2002;288(16):2008-14. doi: 10.1001/jama.288.16.2008.

48. Evans JL, Goldfine ID, Maddux BA, Grodsky GM. Oxidative stress and stress-activated signaling pathways: A unifying hypothesis of type 2 diabetes. Endocr Rev. 2002;23(5):599622. doi: 10.1210/er.2001-0039.

49. Paradis V, Perlemuter G, Bonvoust F, Dargere D, Parfait B, Vidaud $M$, et al. High glucose and hyperinsulinemia stimulate connective tissue growth factor expression: A potential mechanism involved in progression to fibrosis in nonalcoholic steatohepatitis. Hepatology. 2001;34(4 Pt 1):738-44. doi: 10.1053/jhep.2001.28055.

50. Nakahara T, Hyogo H, Yoneda M, Sumida Y, Eguchi Y, Fujii H, et al. Type 2 diabetes mellitus is associated with the fibrosis severity in patients with nonalcoholic fatty liver disease in a large retrospective cohort of japanese patients. J Gastroenterol. 2014;49(11):1477-84. doi: 10.1007/s00535-013-0911-1.

51. Perumpail BJ, Khan MA, Yoo ER, Cholankeril G, Kim D, Ahmed A. Clinical epidemiology and disease burden of nonalcoholic fatty liver disease. World J Gastroenterol. 2017;23(47):82638276. doi: 10.3748/wjg.v23.i47.8263.

52. Summart $U$, Thinkhamrop B, Chamadol N, Khuntikeo N, Songthamwat M, Kim CS. Gender differences in the prevalence of nonalcoholic fatty liver disease in the northeast of Thailand: A population-based cross-sectional study. F1000Res. 2017;6:1630. doi: 10.12688/f1000research.12417.2.

53. Ayonrinde OT, Olynyk JK, Beilin LJ, Mori TA, Pennell CE, de Klerk N, et al. Gender-specific differences in adipose distribution and adipocytokines influence adolescent nonalcoholic fatty liver disease. Hepatology. 2011;53(3):8009. doi: 10.1002/hep.24097.

54. Yang JD, Abdelmalek MF, Pang H, Guy CD, Smith AD, Diehl AM, et al. Gender and menopause impact severity of fibrosis among patients with nonalcoholic steatohepatitis. Hepatology. 2014;59(4):1406-14. doi: 10.1002/hep.26761

55. Seko Y, Sumida Y, Tanaka S, Mori K, Taketani H, Ishiba H, et al. Insulin resistance increases the risk of incident type 2 diabetes mellitus in patients with non-alcoholic fatty liver disease. Hepatol Res. 2018;48(3):E42-51. doi: 10.1111/hepr.12925.

56. Sima A, Timar R, Vlad A, Timar B, Rosu M, Dan I, et al. Nonalcoholic fatty liver disease: a frequent condition in type 2 diabetic patients. Wien Klin Wochenschr. 2014;126(1112):335-40. doi: 10.1007/s00508-014-0530-8

57. Lee HW, Kim KJ, Jung KS, Chon YE, Huh JH, Park KH, et al. The relationship between visceral obesity and hepatic steatosis measured by controlled attenuation parameter. PLoS One. 2017;12(10):e0187066. doi: 10.1371/journal.pone.0187066

58. Zhao H, Song X, Li Z, Wang X. Risk factors associated with nonalcohol fatty liver disease and fibrosis among patients with type 2 diabetes mellitus. Medicine (Baltimore). 2018;97(37):e12356. doi: 10.1097/MD.0000000000012356.

59. Gutierrez-Buey G, Núñez-Córdoba JM, Llavero-Valero M, Gargallo J, Salvador J, Escalada J. Is homa-ir a potential screening test for non-alcoholic fatty liver disease in adults with type 2 diabetes? Eur J Intern Med. 2017;41:74-78. doi: 10.1016/j.ejim.2017.03.006.

60. Wei D, Chen T, Li J, Gao Y, Ren Y, Zhang X, et al. Association of serum gamma-glutamyl transferase and ferritin with the metabolic syndrome. J Diabetes Res. 2015;2015:741731. doi: $10.1155 / 2015 / 741731$. 